\title{
Study of equatorial regions of Moon with the help of backscattering coefficient obtained from LRO data
}

\author{
O P N Calla*, Shubhra Mathur and Monika Jangid \\ International Centre for Radio Science, Jodhpur 342 304, India. \\ ${ }^{*}$ Corresponding author. e-mail: opnc06@gmail.com
}

The Lunar Reconnaissance Orbiter (LRO) has a miniature radio-frequency (Mini-RF) payload, i.e., the Synthetic Aperture Radar (SAR) that has provided very fundamental information about the lunar surface and subsurface which was not known inspite of many manned and unmanned missions. Microwave sensors are used for analyzing the equatorial region of the Moon $\left(60^{\circ} \mathrm{N}\right.$ to $\left.60^{\circ} \mathrm{S}\right)$ which is covered with many well-known craters like Kopff, Taylor, Maunder, Descartes, Jackson and Santos Dumont, each having different topography. The LRO data in terms of the scattering coefficient $\left(\sigma_{\mathrm{LH}}^{\circ}\right.$ and $\left.\sigma_{\mathrm{LV}}^{\circ}\right)$ with incidence angle of $49^{\circ}$ has been used for computing physical and electrical parameter of lunar surface and to learn more about the impact cratering process. Most of the lunar surface shows small Circular Polarization Ratio (CPR), i.e., the reversal of polarization is normal, but some targets have high CPR. In this paper we have discussed the scattering behaviour of lunar equatorial region where the value of CPR $>1$. Studies say that the LV intensity is always greater than LH but from the data obtained from $\mathrm{LRO}$, it is observed that it varies at each pixel depending upon the target properties under radar view.

\section{Introduction}

Microwave remote sensing encompasses both active and passive techniques (Ulaby et al. 1981). Microwave sensors are used as tools for microwave remote sensing and these are of two types - passive and active sensors. Passive sensor detects the selfemissions of the target. Imaging and non-imaging radiometer are the passive sensors. Active sensor measures the scattering coefficient of the target. The active sensors are imaging radars such as the side looking airborne radar (SLAR) or synthetic aperture radar (SAR). The non-imaging active sensors are scatterometer and altimeters.

The microwaves possess some unique capabilities such as (Ulaby et al. 1981):

- Capability to penetrate clouds.
- Independent of Sun as source and so microwave sensors can be used in day time as well as at night.

- Sensitive to the moisture content.

These properties are useful for the study of planets and other heavenly bodies. The Mini-RF onboard LRO (Michael Reid 2010) is a hybrid polarimetric synthetic aperture radar. In this system, a circularly polarized signal is transmitted and the linear vertical and horizontal polarized signals are received by the radar for further processing.

Among radar-based systems, which include scatterometer, altimeters, SAR is commonly used for imaging. SAR (Chan and Koo 2008) uses relative motion between an antenna and its target region and provides distinctive long-term coherent-signal variations that are processed to obtain finer spatial resolution. SAR images have wide applications

Keywords. Remote sensing; lunar surface; circular polarization ratio; scattering coefficient; Stokes vector. 
in remote sensing and are used for mapping terrestrial and planetary surfaces. The SAR-processor stores the radar returned signals, such as amplitude and phase, processes it over successive pulses obtained from elements of synthetic aperture. After a given number of cycles, the stored data is recombined to create a high resolution image of the terrain over which the SAR has collected data.

\section{Hybrid polarimetry and Stokes vector}

A hybrid-polarity SAR (Keith Raney et al. 2011) is one in which transmitted field is circularly polarized, and the resulting backscatter is received in two mutually coherent linear polarizations. Transmission of either right- or left-circular polarization does not matter, as the selection can depend on minimization of gain and phase imbalances in the transmitted field. As long as the relative phase between the received polarizations is retained, the specific choice of linear orientation is insignificant. For convenience, linear polarization is assumed. The complex backscattered signals are transformed into the four Stokes parameters, which are sufficient to capture all of the information conveyed in the backscattered EM field as shown in figure 1.

Polarization (Boerner et al. 1988) has an important role in radar backscattered signal whose intensity depends on target roughness, moisture content and signal characteristics such as incidence angle, wavelength, polarization, etc. Hybrid polarimetry is a polarization technique in which transmitted field is circularly polarized, and the resulting backscatter is received in two mutually coherent linear polarizations. It has proved to be an important tool to calculate the scattering matrix, polarization and Stokes matrix of the received backscattered information.

The Stokes parameters (Sabry and Vachon 2008) are a set of values that describe the polarization state of electromagnetic radiation. The Stokes parameters $S_{0}, S_{1}, S_{2}$ and $S_{3}$ are defined in terms of the amplitude of electric field components of orthogonal states along two distinct axes perpendicular to the direction of propagation. It gives the common description of incoherent or partially polarized radiation in terms of its total intensity $(I)$, degree of polarization $(\delta)$, and the shape parameters of the polarization ellipse. In particular, child parameters, i.e., degree of depolarization, degree of linear polarization and CPR may be determined from the Stokes parameters. The values of these parameters provide objective indications of geophysical features of the lunar surface.

\section{Mini-RF data}

Radar is one of the few remote sensing tools capable of distinguishing water ice from other forms of water that might be present on the Moon. The SAR data (Jawin et al. 2012) is received in the PDS format (Stokes vector). The Mini-RF instrument orbited around the Moon with resolution of $30 \mathrm{~m} /$ pixel and two wavelength bands, a primary band at $12.6 \mathrm{~cm}$ and a secondary band at $4.2 \mathrm{~cm}$.

A strip of LRO contains 16 bytes of data in four channels of four bytes per pixel given by $|\mathrm{LH}|^{2},|\mathrm{LV}|^{2}$, real $\left(\mathrm{LH} \mathrm{LV}^{*}\right)$ and imaginary $\left(\mathrm{LH} \mathrm{LV}^{*}\right)$ where $|\mathrm{LH}|^{2}$ and $|\mathrm{LV}|^{2}$ represent the intensity images for horizontal and vertical received

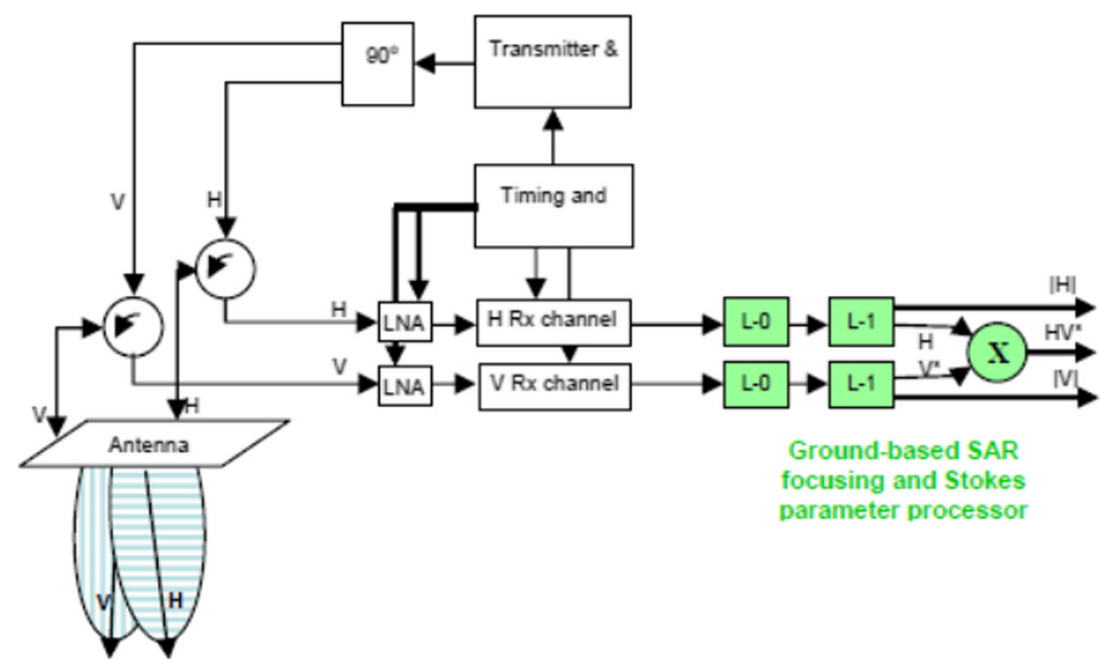

Figure 1. Generic hybrid polarity architecture in which the transmitted field is circularly polarized (right or left), and the receive polarization basis is linear. The data received on the $\mathrm{H}$ and $\mathrm{V}$ arrays are fed to dedicated receiver channels, to get (complex) images, preserving the magnitudes $|\mathrm{H}|$ and $|\mathrm{V}|$, and the relative phase $\mathrm{HV}^{*}$. 
Table 1. Study area.

\begin{tabular}{lllll}
\hline & & & Diameter \\
Sl. no. & \multicolumn{1}{c}{ Crater } & \multicolumn{1}{c}{ Lat./Long. } & $(\mathrm{km})$ & \multicolumn{1}{c}{ Description } \\
\hline 1 & Taylor & $\left(16.7^{\circ} \mathrm{E}, 5.3^{\circ} \mathrm{S}\right)$ & 43 & Old impact crater; tormented floor; central mountain \\
2 & Descartes & $\left(15.7^{\circ} \mathrm{E}, 11.7^{\circ} \mathrm{S}\right)$ & 49 & Damaged circular crater; tormented floor; crest and rille line \\
3 & Maunder & $\left(93.8^{\circ} \mathrm{W}, 14.6^{\circ} \mathrm{S}\right)$ & 55 & Old impact crater; zone of librations \\
4 & Kopff & $\left(89.6^{\circ} \mathrm{W}, 17.4^{\circ} \mathrm{S}\right)$ & 43 & Crater floor flat, filled with lava volcanic origin \\
5 & Jackson & $\left(163.1^{\circ} \mathrm{W}, 22.4^{\circ} \mathrm{N}\right)$ & 71 & Fresh crater \\
6 & Santos Dumont & $\left(4.8^{\circ} \mathrm{E}, 27.7^{\circ} \mathrm{N}\right)$ & 9 & Small circular, impact crater \\
\hline
\end{tabular}
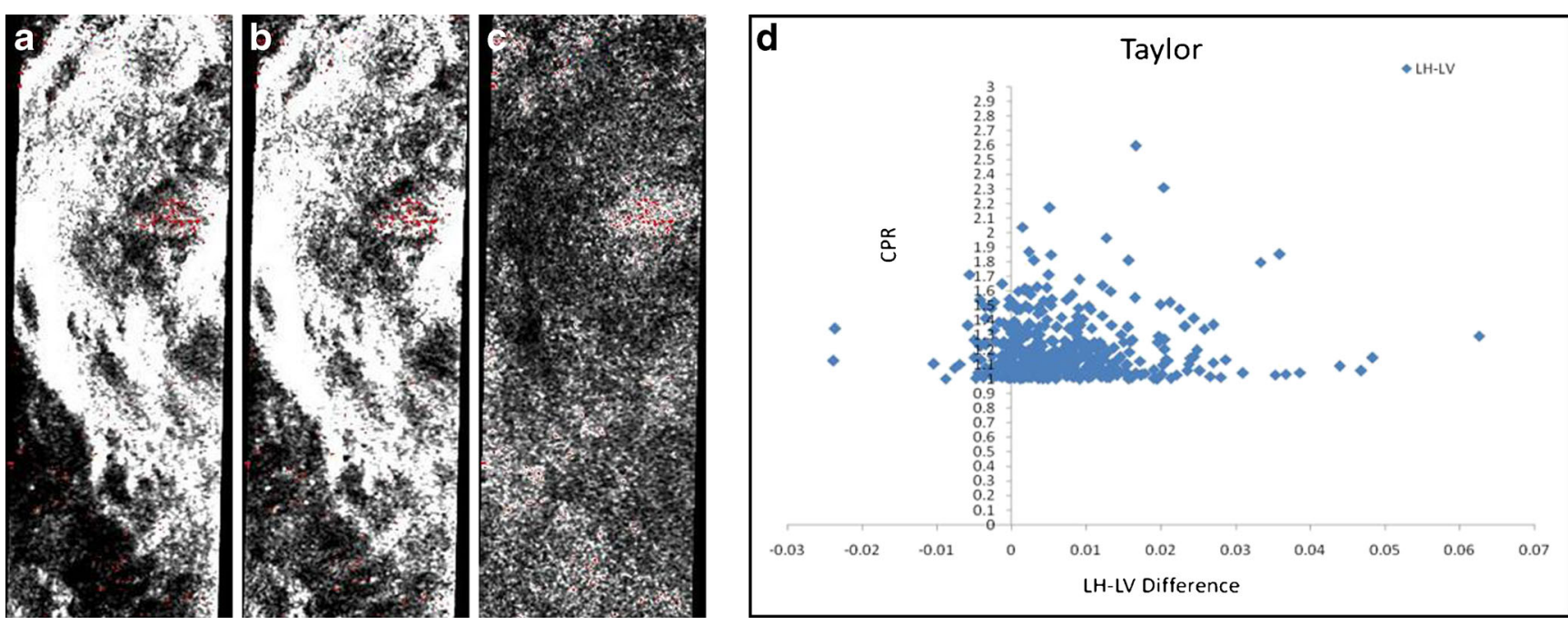

Figure 2. Taylor Crater (a) LH intensity, (b) LV intensity, (c) CPR and (d) CPR vs. LH-LV.

signal, respectively. Real ( $\mathrm{LH} \mathrm{LV}^{*}$ ) and imaginary $\left(\mathrm{LH} \mathrm{LV} \mathrm{LV}^{*}\right.$ represent real and imaginary components of the complex cross power intensity between the horizontal and vertical received signal, respectively. The Stokes vectors generated from the above four parameters are given by equation (1) which describes the polarization state of the received wave.

$$
\left(\begin{array}{l}
\mathrm{S}_{0}=\left\langle\left|\mathrm{E}_{\mathrm{LH}}\right|^{2}+\left|\mathrm{E}_{\mathrm{LV}}\right|^{2}\right\rangle \\
\mathrm{S}_{1}=\left\langle\left|\mathrm{E}_{\mathrm{LH}}\right|^{2}-\left|\mathrm{E}_{\mathrm{LV}}\right|^{2}\right\rangle \\
\mathrm{S}_{2}=2 \mathrm{Re}\left\langle\mathrm{E}_{\mathrm{LH}} \cdot \mathrm{E}_{\mathrm{LV}}^{*}\right\rangle \\
\mathrm{S}_{3}=-2 \operatorname{Img}\left\langle\mathrm{E}_{\mathrm{LH}} \cdot \mathrm{E}_{\mathrm{LV}}^{*}\right\rangle
\end{array}\right),
$$

where $\mathrm{E}_{\mathrm{LH}}=$ Electric field for horizontal linear polarization, $\mathrm{E}_{\mathrm{LV}}=$ Electric field for vertical linear polarization, and ' $*$ ' = Represents the conjugate of the complex number.

\section{Circular polarization ratio (CPR)}

$\mathrm{CPR}$ is defined as the ratio of received power in the same sense (SC) transmitted to the opposite sense (OC) (Saran et al. 2012). It is one of the parameters derived from Stokes vector. The CPR $(\mu)$ is the most important parameter, as it provides the indications for wavelength-scale complexity of the surface (Stewart Nozette et al. 2001). When an incident beam reflects off a surface, it changes the sense of polarization opposite of the signal transmitted (OC); likewise, if the beam reflects off two objects, it reverses polarization twice, therefore resulting in the same sense in which it was transmitted (SC).

The CPR analysis over selected craters shows how the variations of the different parameters affect the CPR ratio, indicating reasons for the variations in the observed data. Rough, rocky surface causes incident radar beams to bounce more than once, leading to more SC signals and thus high CPR; therefore, rougher surfaces show higher mean CPR. Depending on the radar wavelength and the composition of the regolith, it is possible to observe features below the surface which may be due to the surface roughness or ejecta of the lava flows on the lunar floor. This is due to two reasons, firstly melt deposits came from oblique impacts that are capable of ejecting melted material farther downrange and secondly it may 

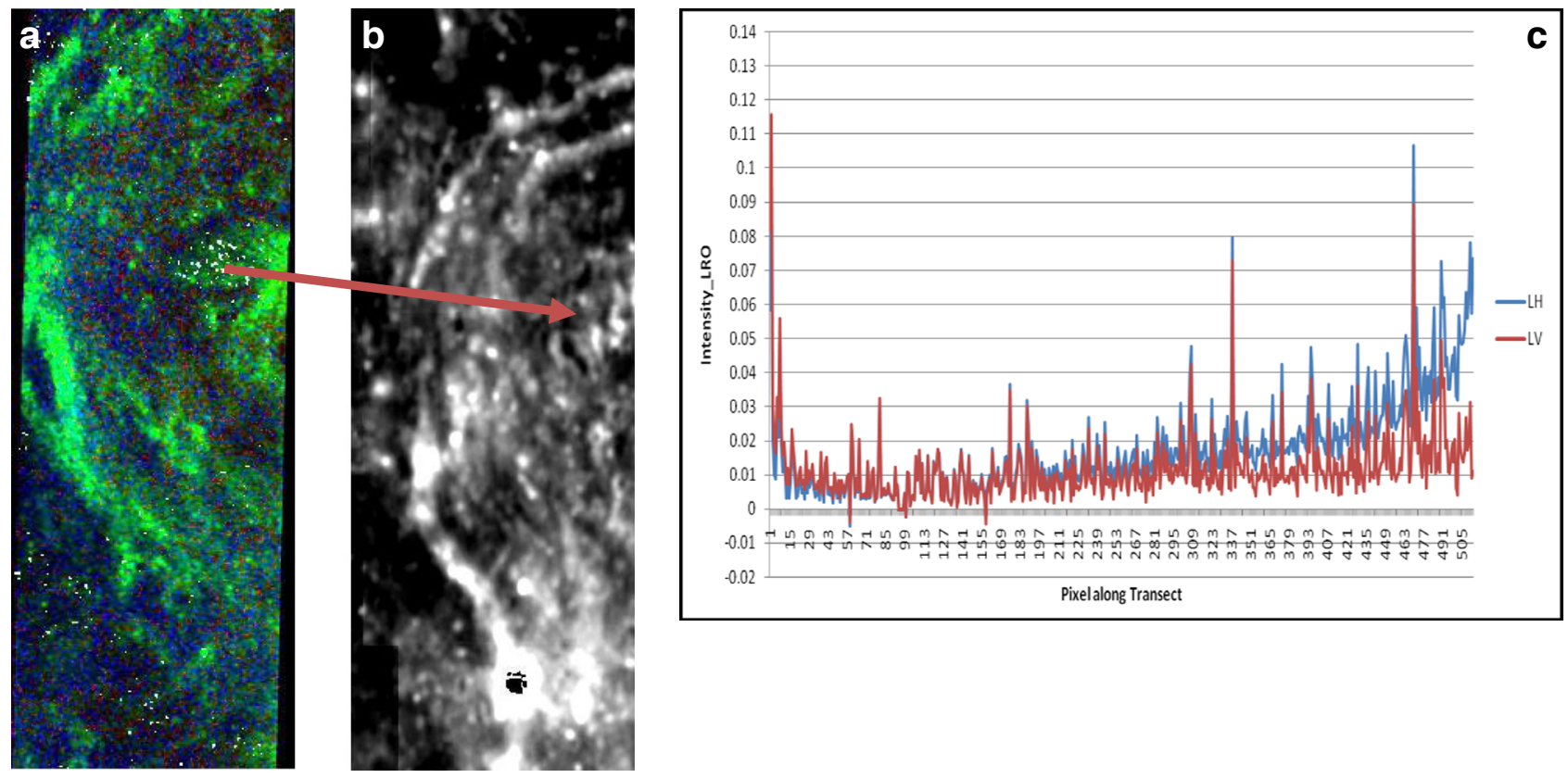

Figure 3. (a) m-chi decomposition with CPR values, (b) optical image, (c) variation in LH and LV intensity using LRO data in Taylor Crater.
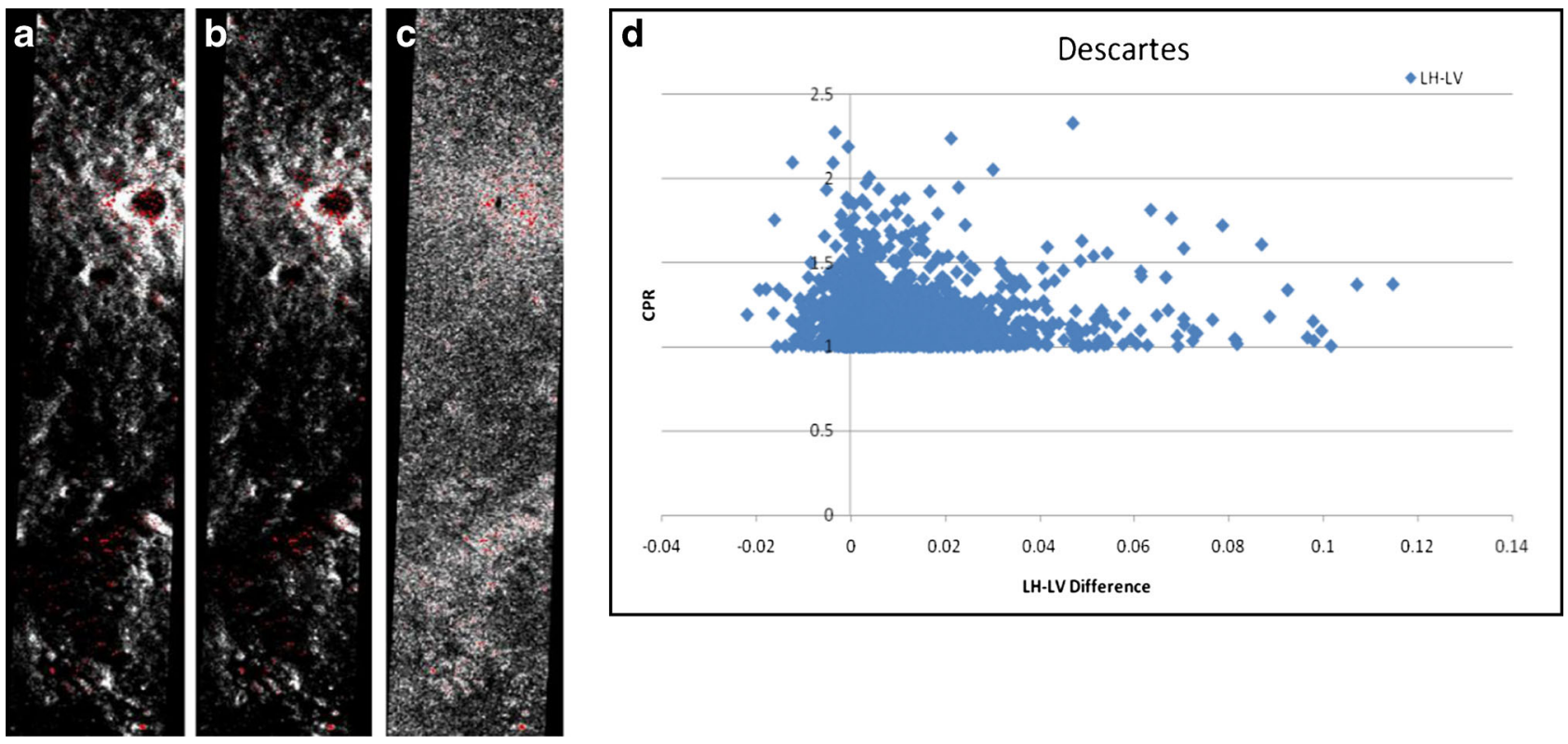

Figure 4. Descartes Crater (a) LH intensity, (b) LV intensity, (c) CPR and (d) CPR vs. LH-LV.

also be associated with older, nearby craters that no longer have a radar-bright proximal ejecta blanket. The CPR range varies between 0 and 2.5 in the equatorial region of the Moon. Changes in backscatter values along many of the ejecta flows are probably caused by features typical of lava flows. The CPR can be calculated by the following formulation as given by equation (2)

$$
\mathrm{CPR}=\mathrm{SC} / \mathrm{OC}=\left(\mathrm{S}_{0}-\mathrm{S}_{3}\right) /\left(\mathrm{S}_{0}+\mathrm{S}_{3}\right) .
$$

\section{Study area}

The present study has been carried out using the S-band data of the LRO Mini-RF. Impact craters possess dominant geographic features on the Moon (Dieter Stöffler et al. 2006). Simple impact craters have bowl-shaped depressions, mostly with smooth walls while complex impact craters have a single or multiple peaks in the middle of the crater. During analysis we have tried to cover all the possible 

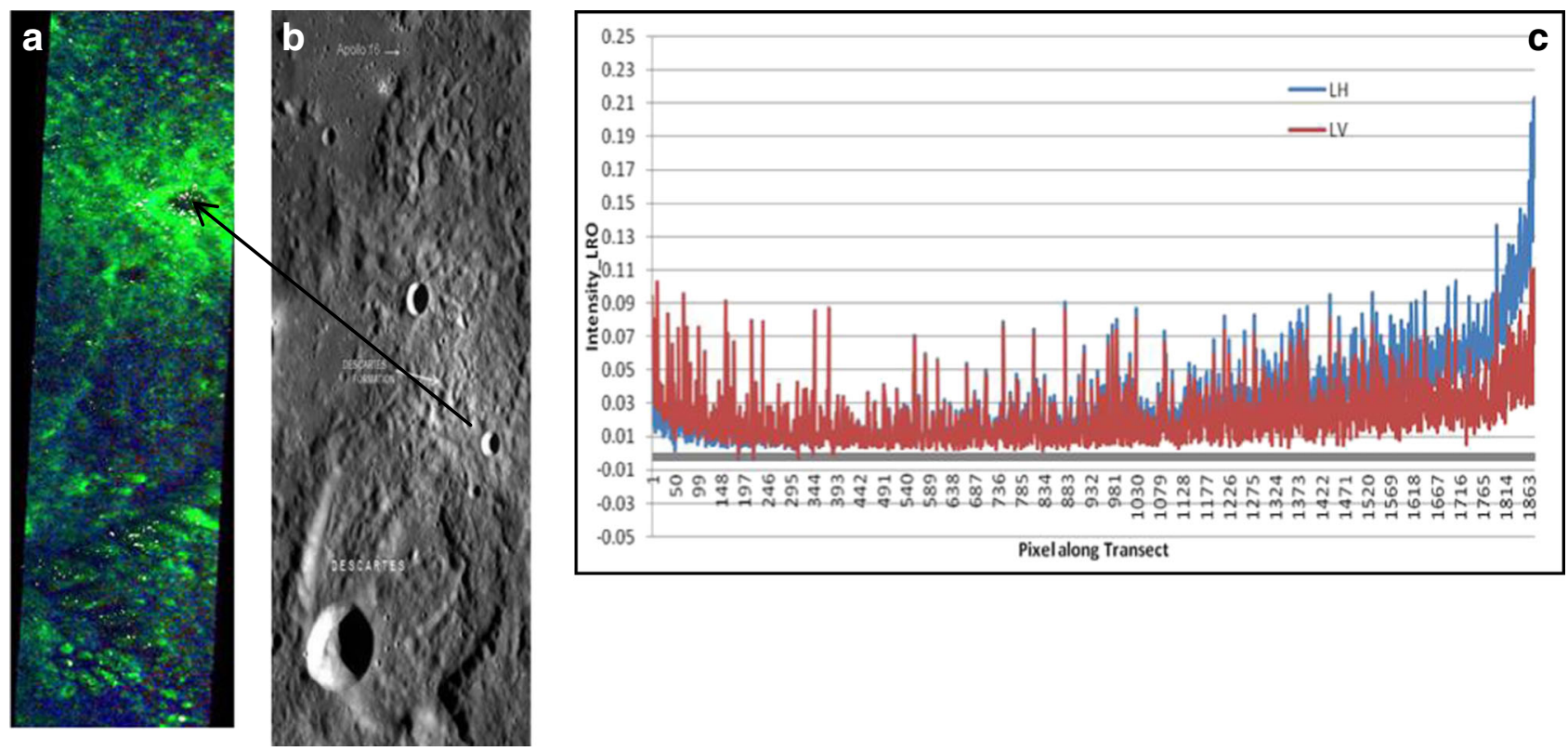

Figure 5. (a) m-chi decomposition with CPR values, (b) optical image and (c) variation in LH and LV intensity using LRO data in Descartes Crater.
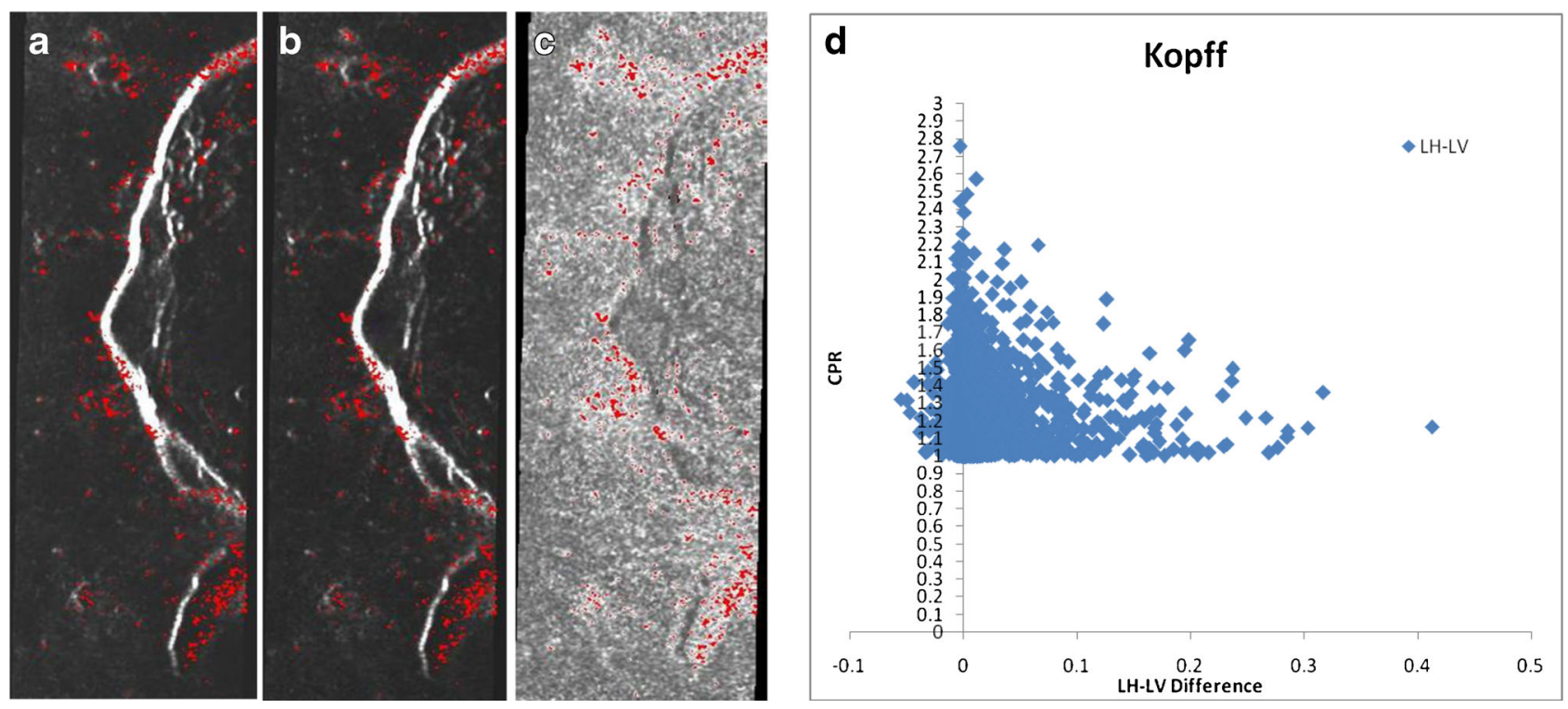

Figure 6. Kopff Crater (a) LH intensity, (b) LV intensity, (c) CPR and (d) CPR vs. LH-LV.

craters on the equatorial region. The LRO strips at an incidence angle $\sim 49^{\circ}$ provide the details of the lunar features. These features include craters which are listed in table 1. Among these features, the Taylor and Descartes Craters belong to the lunar highland region, while the Maunder and Kopff Craters are part of the Mare Orientale basin on the lunar near side. Santos Dumont Crater lies in the Montes Apenninus range at the eastern edge of the Mare Imbrium basin. The lunar far side crater Jackson was also included in this study, which is a fresh crater surrounded by a system of rays created by ejecta.

\section{Results and discussion}

\section{1 m-Chi decomposition}

This decomposition is based on the degree of polarization $(\mathrm{m})$ and the poincare ellipticity parameter (chi). Analysis of the equatorial craters demonstrates how m-chi (Keith Raney et al. 2012) 

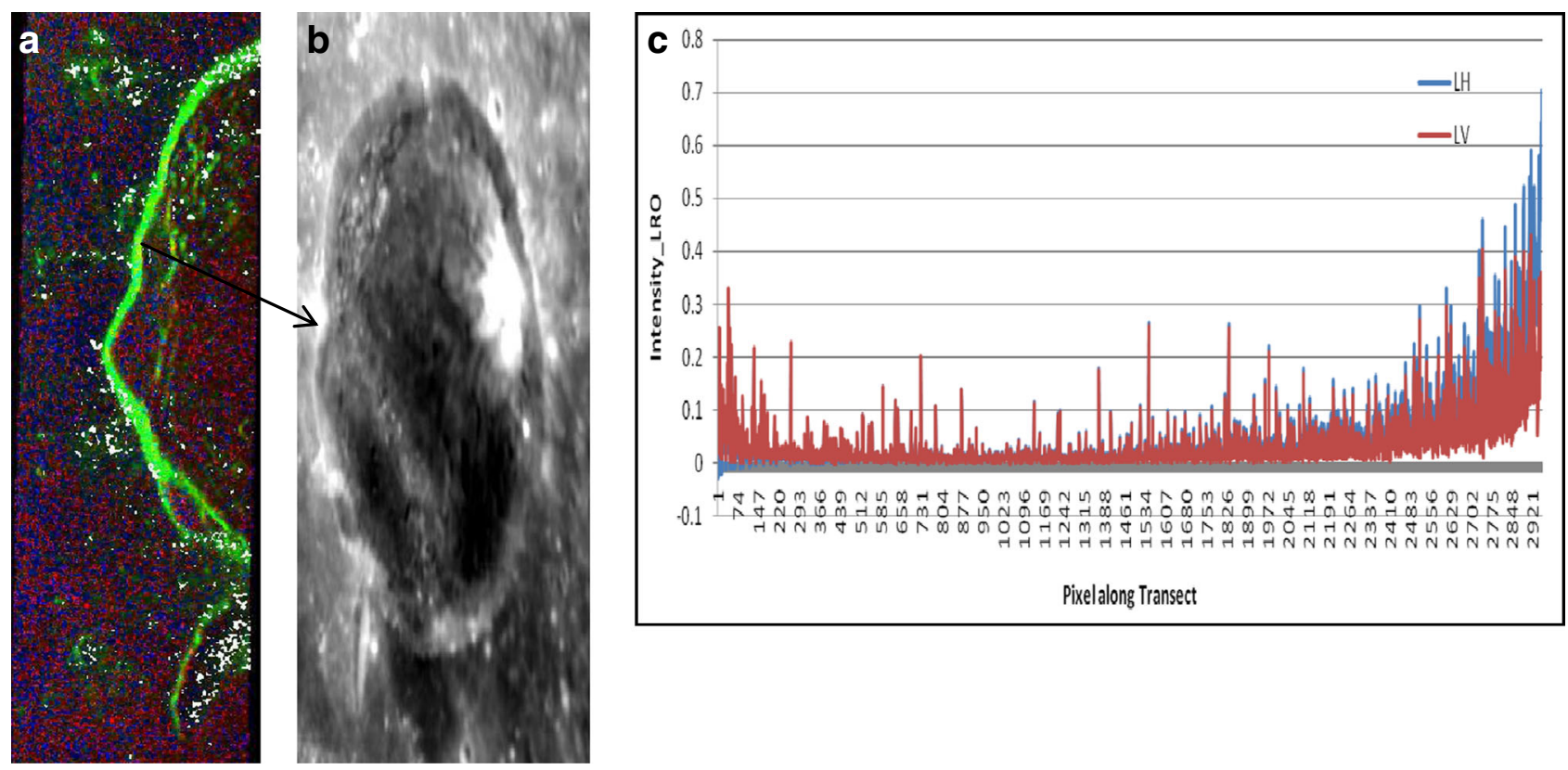

Figure 7. (a) m-chi decomposition with CPR values, (b) optical image and (c) variation in LH and LV intensity using LRO data in Kopff Crater.
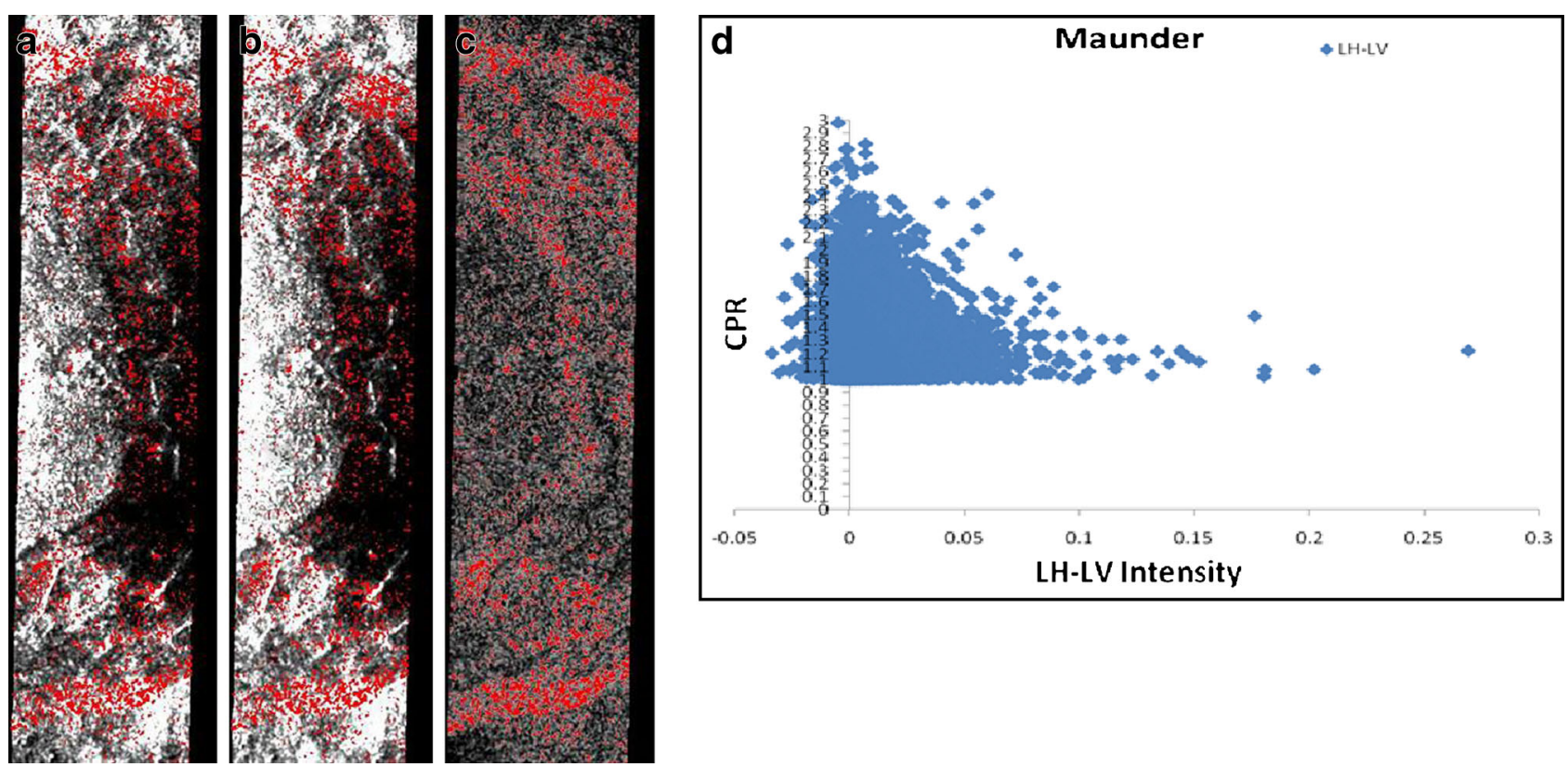

Figure 8. Maunder Crater (a) LH intensity, (b) LV intensity, (c) CPR and (d) CPR vs. LH-LV.

can more easily differentiate materials within ejecta deposits and their relative thicknesses. m-chi decomposition consists of the scattering like surface scattering, double bounce and volume scattering. The $\mathrm{m}$-chi values in the maps are represented using different colours explained below:

- Blue colour indicates single bounce scattering (if an incident wave with a particular polarization, has a simple interaction with a target, i.e., lunar regolith).
- Red corresponds to double bounce that might occur between two surfaces at an angle to one another (due to presence of bedrocks beneath the surface).

- Green colour represents the randomly polarized constitutes or volume scattering (the incident wave undergoes many bounces before returning to the radar).

The white points on m-chi images show the CPR $>1$. 

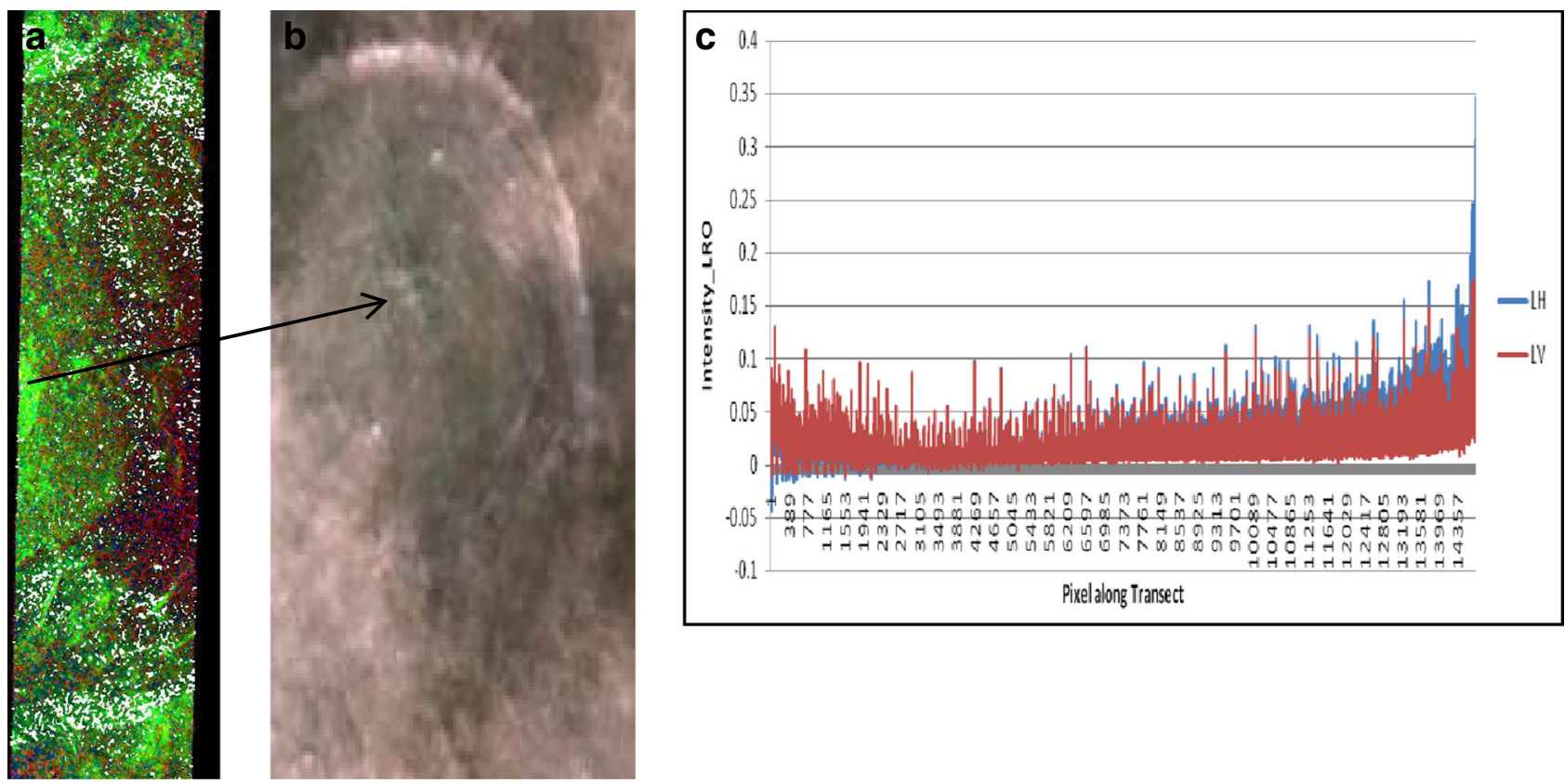

Figure 9. (a) m-chi decomposition with CPR values, (b) optical image and (c) variation in LH and LV intensity using LRO data in Maunder Crater.
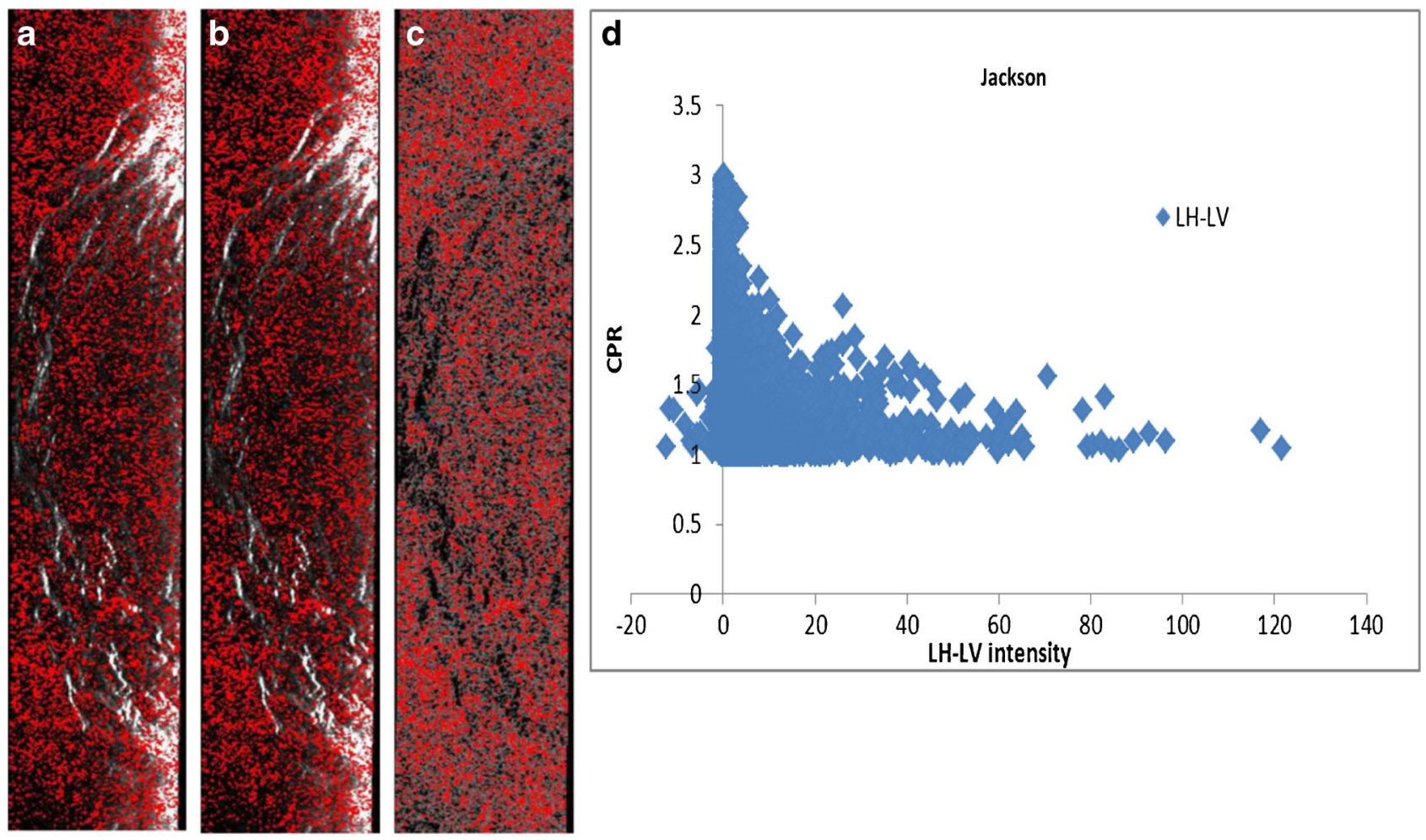

Figure 10. Jackson Crater (a) LH intensity, (b) LV intensity, (c) CPR and (d) CPR vs. LH-LV.

\subsection{Taylor and Descartes Crater}

The maximum volume scattering was observed on the Taylor Crater terraces and central peak as shown in figures 2 and 3 . The presence of small craters on the floor and worn edges of the crater rim have surface scattering and double bounce scattering. The high values of CPR are observed on the central peak and near the crater's rim.

Descartes is a heavily worn lunar crater. Volume scattering can be seen at damaged circular formation with terraced walls composed of several 

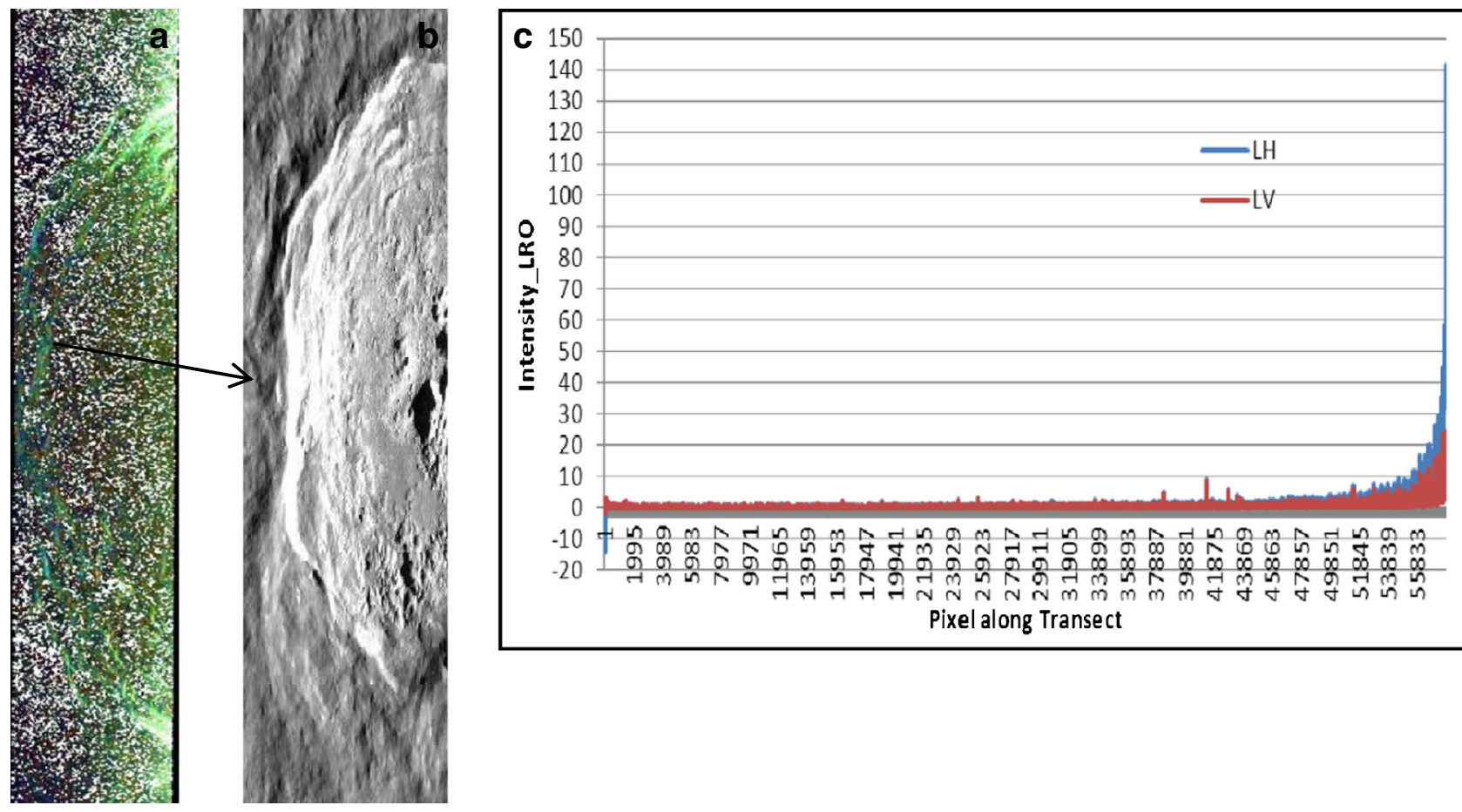

Figure 11. (a) m-chi decomposition with CPR values, (b) optical image and (c) variation in LH and LV intensity using LRO data in Jackson Crater.
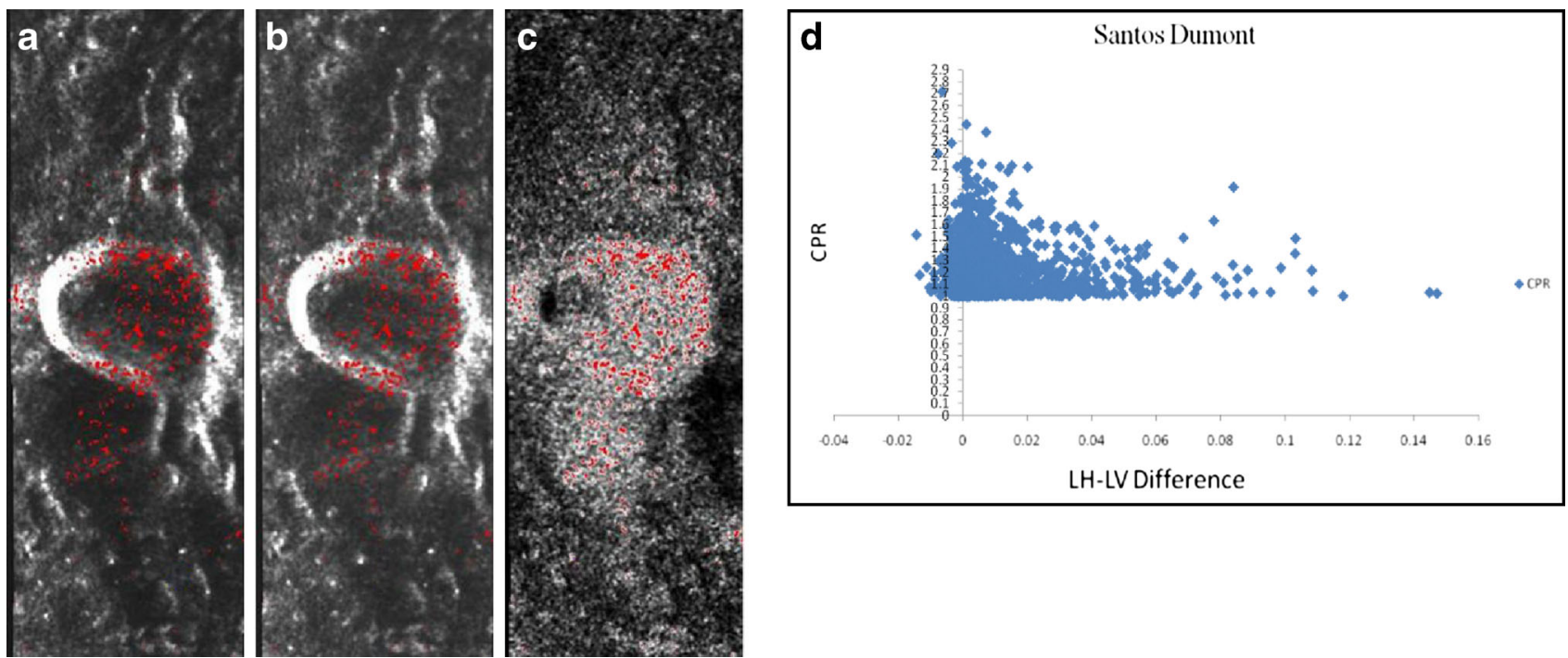

Figure 12. Santos Dumont (a) LH intensity, (b) LV intensity, (c) CPR and (d) CPR vs. LH-LV.

concentric rings because of scattered ejecta. It holds same properties as that of Taylor Crater. Even due to the presence of the highlands and ejecta deposit, the floor shows surface scattering because they have been filled by the melted lava from the other impacts as shown in figures 4 and 5 . The higher CPR values $(>1)$ are observed due to the double bounce scattering or due to the lava flow which comprised of the small size particle rocks, i.e., volume scattering.

\subsection{Kopff and Maunder Crater}

Kopff Crater has an unusual rim shape and ejecta deposits with no central peak and no wall terraces. Secondary crater are also present on its floor. Crater's rim and ejecta deposits show the volume scattering. Double bounce scattering is dominant on the crater floor where cracks are present which is because of upliftment in the crater floor. The values of $\mathrm{CPR}(>1)$ are observed near rim of the 

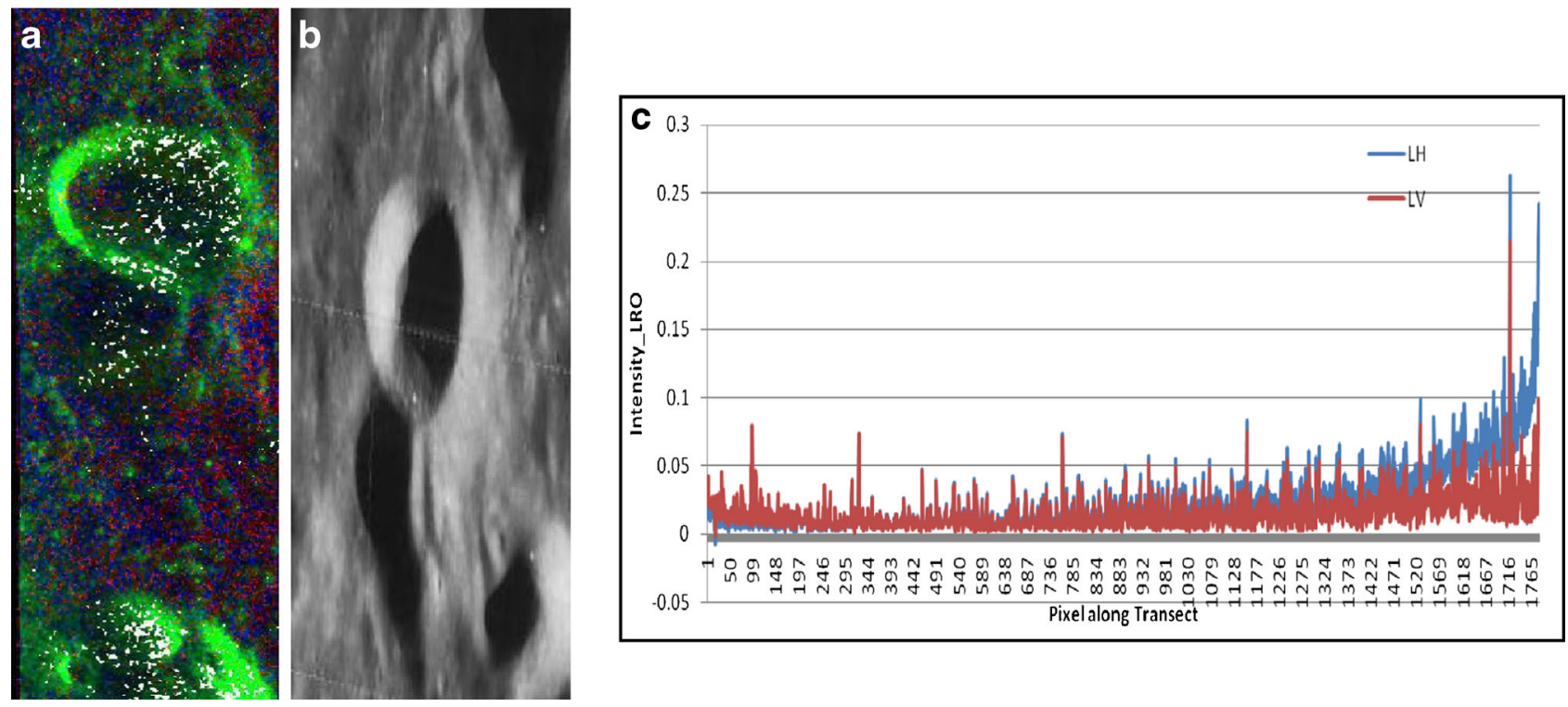

Figure 13. (a) m-chi decomposition with CPR values, (b) optical image and (c) variation in LH and LV intensity using LRO data in Santos Dumont Crater.

Table 2. Comparison table of craters with scattering coefficient having $C P R>1$.

\begin{tabular}{|c|c|c|c|c|}
\hline Sl. no & Crater name & Parameters & $\begin{array}{l}\text { Total points observed } \\
\quad(\text { where } \mathrm{CPR}<1 \text { ) }\end{array}$ & CPR (avg.) \\
\hline \multirow{3}{*}{1} & \multirow{3}{*}{ Taylor } & $\mathrm{LH}>\mathrm{LV}$ & 99 & 1.147 \\
\hline & & $\mathrm{LH}=\mathrm{LV}$ & 7 & 1.117 \\
\hline & & $\mathrm{LH}<\mathrm{LV}$ & 407 & 1.186 \\
\hline \multirow{3}{*}{2} & \multirow{3}{*}{ Descartes } & $\mathrm{LH}>\mathrm{LV}$ & 373 & 1.176 \\
\hline & & $\mathrm{LH}=\mathrm{LV}$ & 12 & 1.137 \\
\hline & & $\mathrm{LH}<\mathrm{LV}$ & 1490 & 1.157 \\
\hline \multirow{3}{*}{3} & \multirow{3}{*}{ Kopff } & $\mathrm{LH}>\mathrm{LV}$ & 746 & 1.248 \\
\hline & & $\mathrm{LH}=\mathrm{LV}$ & 17 & 1.213 \\
\hline & & $\mathrm{LH}<\mathrm{LV}$ & 2194 & 1.201 \\
\hline \multirow{3}{*}{4} & \multirow{3}{*}{ Maunder } & $\mathrm{LH}>\mathrm{LV}$ & 3556 & 1.232 \\
\hline & & $\mathrm{LH}=\mathrm{LV}$ & 142 & 1.210 \\
\hline & & $\mathrm{LH}<\mathrm{LV}$ & 11045 & 1.228 \\
\hline \multirow{3}{*}{5} & \multirow{3}{*}{ Jackson } & $\mathrm{LH}>\mathrm{LV}$ & 4460 & 1.307 \\
\hline & & $\mathrm{LH}=\mathrm{LV}$ & 58 & 1.296 \\
\hline & & $\mathrm{LH}<\mathrm{LV}$ & 53300 & 1.265 \\
\hline \multirow{3}{*}{6} & \multirow{3}{*}{ Santos Dumont } & $\mathrm{LH}>\mathrm{LV}$ & 327 & 1.188 \\
\hline & & $\mathrm{LH}=\mathrm{LV}$ & 14 & 1.173 \\
\hline & & $\mathrm{LH}<\mathrm{LV}$ & 1441 & 1.196 \\
\hline
\end{tabular}

crater, the floor of the ejecta and exterior of the crater because of roughness as shown in figures 6 and 7 .

Maunder Crater has the similar topography like Taylor Crater. Multilayer surface of Maunder Crater signifies volume scattering at central peak and places where continuous ejecta deposits are present. The rim of Maunder is roughly circular, with the terraced inner wall as shown in figures 8 and 9. Terraces are not eroded and due to this it shows double bounce scattering. Terrace wall which was formed due to the ejecta or presence of rock particles makes CPR $(>1)$ higher at these places.

\subsection{Jackson and Santos Dumont}

Jackson is a fresh crater located on the far side of the northern hemisphere. It lies at the center 
of large ray system. This crater has a number of terraces moving downward into the crater floor, backscattering and CPR values vary accordingly. The RGB image shows the maximum volume scattering at the interior region of the crater because of the presence of buried rock materials; while the terrace and floor of the crater give some points of double bounce and surface scattering. Very rough floor causes high CPR values, formed due to gradual cooling of post-impact melt material. The extreme ends of the crater are very bright due to the presence of excessive amounts of rock deposits giving very high backscattered values as shown in figures 10 and 11.

Santos Dumont is an impact crater, i.e., small, circular and bowl-shaped in size with ejecta deposits present on the crater floor. It has very low CPR on the western rim of the crater. The high values of $C P R$ can be seen within the interior of the crater as shown in figures 12 and 13 which is due to moderate rough floor of the crater. The decomposition techniques differentiate the scattering mechanism in different areas. Volume scattering is dominant scattering mechanism on the crater rim. The interior floor of crater is being characterized with double bounce and surface scattering.

The overall observation of all these craters is summarized in table 2 .

\section{Conclusion}

Radar backscattering properties and CPR variation in the equatorial craters like Taylor, Kopff, Maunder, Jackson, Descartes and Santos Dumont have been studied using Mini-RF data of LRO. The LRO data provides data strips with the maximum points having LV intensity greater than LH intensity. The likely reasons for this behaviour could be the slope of the topography along the look direction and surface composition (Samuel et al. 2011). When the slope is angled towards the spacecraft, more radar energy is scattered in the direction of SAR and vice versa. Secondly, the specific composition of the target material also plays an important role related to scattering from the target and scattered energy received by SAR. At the same time, there are some points on the surface from where the scattered energy received by SAR does not vary to a large extent because of the non-variability of composition of the target material. (Note: For surface scattering condition: $|\mathrm{LV}|^{2}>|\mathrm{LH}|^{2}$.)

In this paper we have considered only those points where CPR is greater than 1 since lunar surface roughness varies, and this roughness increases the CPR value as high as 1 . However, CPR higher than 1 can be observed in two situations: (i) the geometry of a crater is such that large amount of energy is scattered back to the receiver either by bouncing first off the floor of the crater and secondly off the wall or vice versa. These doublebounce returns yield CPR values so high that they can sometimes exceed 1. (ii) Surface morphology, i.e., due to the presence of lava deposits, small size rock particles, terraces, etc., point to volume and double bounce scattering which in turn increases the CPR.

The values of CPR $>1$ is attributed to the rough surface, which is further confirmed from the comparison of CPR obtained at different location on the same crater. The high value of $\mathrm{CPR}>1$ is from the location where lava deposits have created a rough surface while the $\mathrm{CPR}<1$ is obtained from the comparatively smoother surface. m-chi decomposition techniques help in identifying crater morphology and understanding CPR values over lunar surface.

\section{Acknowledgements}

Authors would like to acknowledge PLANEX PRL, India, Prof J N Goswami, Director, PRL and Dr S V S Murthy, Senior Professor and Coordinator PLANEX PRL, Navrangpura, Ahmedabad and SAC, ISRO India for their support. They thank NASA team for providing their respective LRO data for analysis and ICRS staff for the support and help.

\section{References}

Boerner W M, Mott H, Lunenburg E, Livingstone C, Brisco B, Brown R J and Patterson J S 1998 Polarimetry in remote sensing: Basic and applied concepts; chapter 5, In: Manual of Remote Sensing, Vol. 2, Principles and Applications of Imaging Radar, 3rd edn, Wiley.

Chan Y K and Koo 2008 An introduction to synthetic aperture radar (SAR); Progr. Electromag. Res. B 2 27-60.

Dieter Stöffler, Graham Ryder, Boris A Ivanov, Natalia A Artemieva, Mark J Cintala and Richard A F Grieve 2006 Cratering history and lunar chronology; Rev. Mineral. Geochem. 60 519-596.

Jawin E R, Kiefer W S, Bussey B, Cahill J T, Dyar M D, Fassett C I, Lawrence S and Spudis P D 2012 The relationship between radar scattering and surface roughness of lunar volcanic domes; Lunar and Planetary Science XLIII, Abstract \#1333.

Keith Raney R, Cahill Joshua T S, Wesley Patterson G, Benjamin D and Bussey J 2012 The m-chi decomposition of hybrid dual-polarimetric radar data with application to lunar craters; J. Geophys. Res. 117 1991-2012.

Keith Raney R, Paul Spudis, Ben Bussey, Robert J, Jensen Bill Marinelli, Priscilla McKerracher, Ron Schulze, Herman Sequeira and Helene Winters 2011 Compact polarimetry at the Moon: The Mini-RF radars; POLinSAR, Frascati, Italy.

Michael Reid 2010 PDS Archive Volume: Software Interface Specification (SIS) for Mini-RF Advanced Technologies - Lunar Reconnaissance Orbiter (LRO) Payload Operations; Center prepared by Mini-RF Program. 
Sabry R and Vachon P W 2008 Advanced Polarimetric Synthetic Aperture Radar (SAR) And Electro-Optical (EO) Data Fusion Through Unified Coherent Formulation of the Scattered EM Field; Progr. Electromag. Res. PIER 84 189-203.

Samuel W Bell 2011 Fresh lunar crater ejecta as revealed by the Miniature Radio Frequency (Mini-RF) instrument on the Lunar Reconnaissance Orbiter, Department of Astronomy of Amherst College.

Sriram Saran, Anup Das, Shiv Mohan and Manab Chakraborty 2012 Study of scattering characteristics of lunar equatorial region using Chandrayaan-1 Mini-DSAR polarimetric data; Planet. Space Sci. 71(1) $18-30$.

Stewart Nozette, Spudis Paul D, Robinson Mark S, Bussey D B J and Chris Lichtenbergand Robert Bonner 2001 Integration of lunar polar remote-sensing data sets: Evidence for ice at the lunar south pole; J. Geophys. Res. 106(E10) 23,253-23,266.

Ulaby Fawwaz T, Richard K More and Adrian K Fung 1981 Microwave remote sensing - Active and Passive, Artech House Inc., Vol. 1-3. 